The Astrophysical Journal, 279:827-838, 1984 April 15

(C) 1984. The American Astronomical Society. All rights reserved. Printed in U.S.A.

\title{
ELEMENTAL COMPOSITION OF SOLAR ENERGETIC PARTICLES
}

\author{
W. R. Cook, E. C. Stone, And R. E. Vogt \\ California Institute of Technology \\ Received 1983 June 27; accepted 1983 October 3
}

\begin{abstract}
The Low Energy Telescopes on the Voyager spacecraft have been used to measure the elemental composition $(2 \leq Z \leq 28)$ and energy spectra $(5-15 \mathrm{MeV}$ per nucleon) of solar energetic particles (SEPs) in seven large flare events. Four flare events were selected which have SEP abundance ratios approximately independent of energy per nucleon. For these selected flare events, SEP composition results may be described by an average composition plus a systematic flare-to-flare deviation about the average. The four-flare average SEP composition is systematically different from the solar composition determined by photospheric spectroscopy. These systematic composition differences are apparently not due to SEP propagation or acceleration effects. In contrast, the four-flare average SEP composition is in agreement with measured solar wind abundances and with a number of recent spectroscopic coronal abundance measurements. These findings suggest that SEPs originate in the corona, and that both SEPs and the solar wind sample a coronal composition which is significantly and persistently different from that measured for the photosphere. Our observations thus provide a measure of the coronal abundances of 15 elements.
\end{abstract}

Subject headings: cosmic rays: abundances - Sun: abundances - Sun: corona - Sun: flares Sun: solar wind

\section{INTRODUCTION}

The energetic particles ejected from solar flares constitute a sample of solar material which may yield valuable information on the elemental and isotopic composition of the solar atmosphere - information that impacts a wide range of astrophysical problems from the history of the solar system, to solar structure and dynamics, to nucleosynthesis. Although the composition of the Sun has been extensively studied (see, e.g., the spectroscopic analysis by Russell 1929, and the papers by Claas 1951; Goldberg, Muller, and Aller 1960), the knowledge of solar composition remains inadequate. None of the current techniques to investigate solar composition are free of potential systematic uncertainty. Spectroscopic abundance determinations depend on models of the temperature and density structure of the solar atmosphere and on detailed knowledge of the spectral-line formation mechanisms and atomic transition probabilities. Furthermore, spectroscopic abundances are unavailable for some elements-most notably helium, the second most abundant solar constituent. Similarly, relating the composition of solar wind and energetic flare particles to the composition of the Sun requires a careful assessment of possible acceleration and propagation biases.

The potential of solar energetic particle (SEP) measurements as a source of solar composition information was first explored in the early 1960s using rocket-borne nuclear emulsion experiments with energy thresholds near $40 \mathrm{MeV}$ per nucleon. The early work (see the reviews by Biswas and Fichtel 1965; Bertsch, Fichtel, and Reames 1972) suggested that, for nuclei with nearly the same nuclear charge to mass ratio, the SEPs might represent an unbiased sample of solar material. However, later studies have indicated that, even for elemental species with the same nuclear charge to mass ratios, the SEP elemental composition varies from flare to flare (see, e.g., Armstrong and Krimigis 1971; Armstrong et al. 1972; Mogro-Campero and Simpson 1972; Bertsch et al. 1973; Teegarden, von Rosenvinge, and McDonald 1973; Crawford et al. 1975; Bertsch and Reames 1977; Dietrich and Simpson 1978; McGuire, von Rosenvinge, and McDonald 1979). In some flare events the composition varies with time, space, and/or energy per nucleon (see, e.g., Bertsch, Biswas, and Reames 1974; Van Allen, Venketarangan, and Venkatesan 1974; Crawford et al. 1975; Armstrong et al. 1976; O'Gallagher et al. 1976; Scholer et al. 1976). Some of the more extreme SEP elemental composition anomalies are associated with large ${ }^{3} \mathrm{He}$ enhancements (Hovestadt et al. 1975; Hurford et al. 1975; Zwickl et al. 1978). This apparent complexity and the availability of high-quality SEP composition measurements for only a small number of flare events have made it difficult to determine which features of SEP composition are the same from flare to flare and which are variable.

In this paper we undertake a systematic analysis of SEP composition measurements for seven large flare events with the purpose of determining which properties of the composition vary and which are typical from flare to flare. The individual element resolution and high statistical precision of the measurements have allowed the study of abundances for 15 different elemental species from $\mathrm{He}$ through Ni. For the first time, statistically accurate abundances for the rare elements $\mathrm{Na}, \mathrm{Al}, \mathrm{S}, \mathrm{Ar}, \mathrm{Ca}, \mathrm{Cr}$, and $\mathrm{Ni}$ were obtained for a relatively large number of individual flare events.

Brief accounts of this work have been published earlier (see Cook et al. 1979; Cook, Stone, and Vogt 1980). In addition Mewaldt (1980) reviewed progress in SEP elemental and isotopic composition measurements, including preliminary results of this work. Detailed discussions of the experiment calibration and data analysis are given in Cook (1981). 


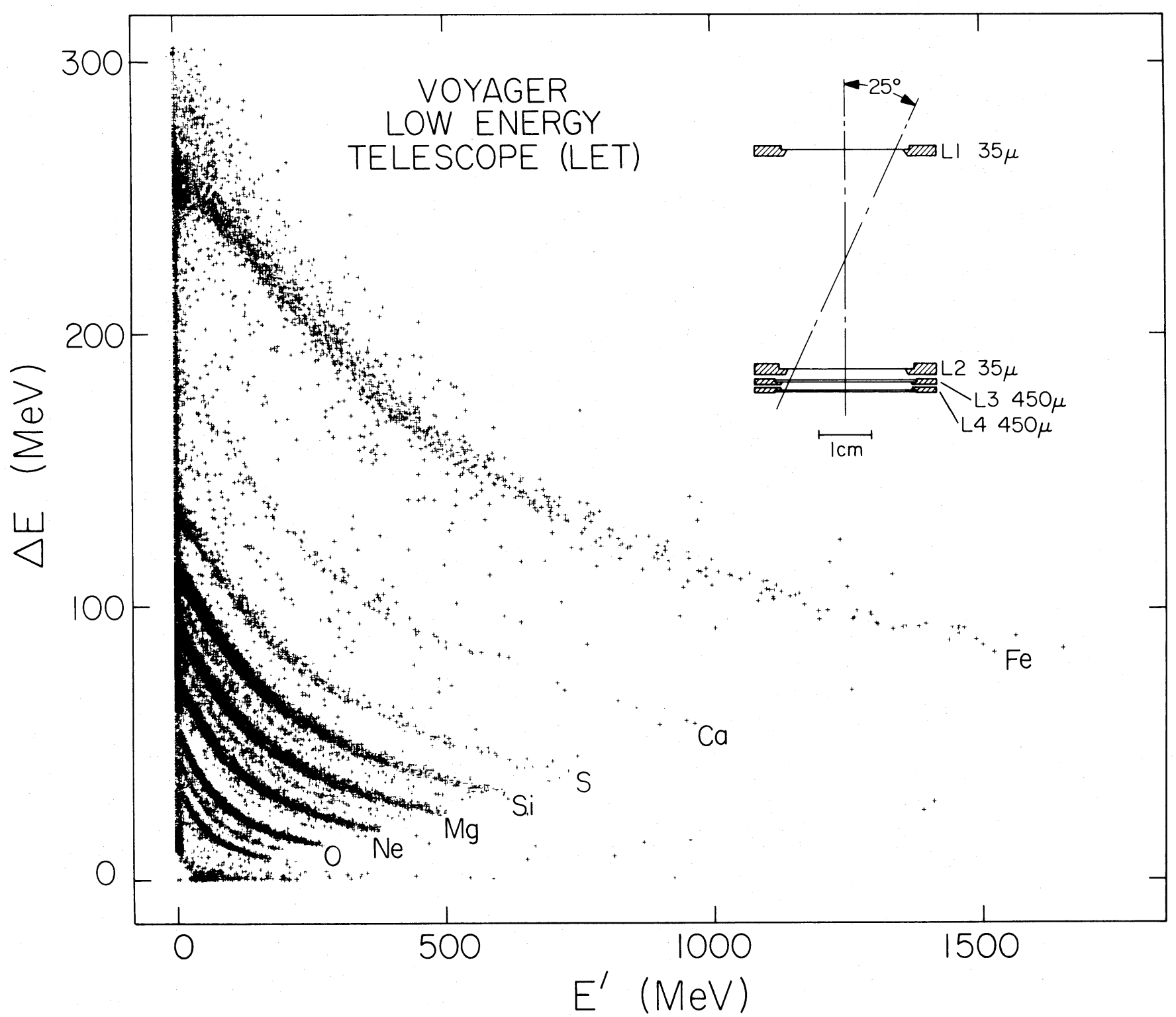

FIG. 1.-LET schematic drawing and a scatter plot of raw flare data. The energy $(\Delta E)$ deposited in L1 is plotted vs. the sum $\left(E^{\prime}\right)$ of energies deposited in detectors L2 and L3 for a sample of $Z \geq 3$ events from a LET on Voyager 1. (To prevent plot saturation, only every tenth event was plotted for elements oxygen and below.)

\section{THE EXPERIMENT}

The observations were made with the Low Energy Telescopes (LETs, described in detail by Stone et al. 1977) in the Cosmic Ray Subsystems on the Voyager 1 and Voyager 2 spacecraft. The LET systems on each spacecraft incorporate four nominally identical charged-particle telescopes which use the differential energy loss (multiple $d E / d x$ ) and total energy $(E)$ technique to measure the kinetic energy and the nuclear charge $Z$ of individual incident nuclei in the range $1 \leq Z \leq 28$. The kinetic energy range of response varies from about $1.8-8 \mathrm{MeV}$ per nucleon for protons and helium nuclei to about 5-30 MeV per nucleon for iron nuclei. The four telescopes provide a relatively large combined geometry factor of $1.7 \mathrm{~cm}^{2} \mathrm{sr}$ and are oriented at orthogonal viewing angles to give three-dimensional information on energetic particle streaming patterns.

Each LET (see the schematic cross section in Fig. 1) contains four totally depleted silicon surface-barrier detectors, labeled L1-L4. Detectors L1, L2, and L3 are pulse height analyzed for particles which trigger the $200 \mathrm{keV}$ thresholds of detectors L1 and L2 but do not trigger the $300 \mathrm{keV}$ threshold of detector L4. Thus, for nuclei which penetrate $\mathrm{L} 1$ and stop in L2 we obtain a $d E / d x$ energy loss measurement from $\mathrm{L} 1$ and a residual energy measurement from $\mathrm{L} 2$. For nuclei which penetrate both $\mathrm{L} 1$ and $\mathrm{L} 2$, we obtain redundant $d E / d x$ measurements from $\mathrm{L} 1$ and $\mathrm{L} 2$ and a residual energy measurement from L3. The additional $d E / d x$ measure- 


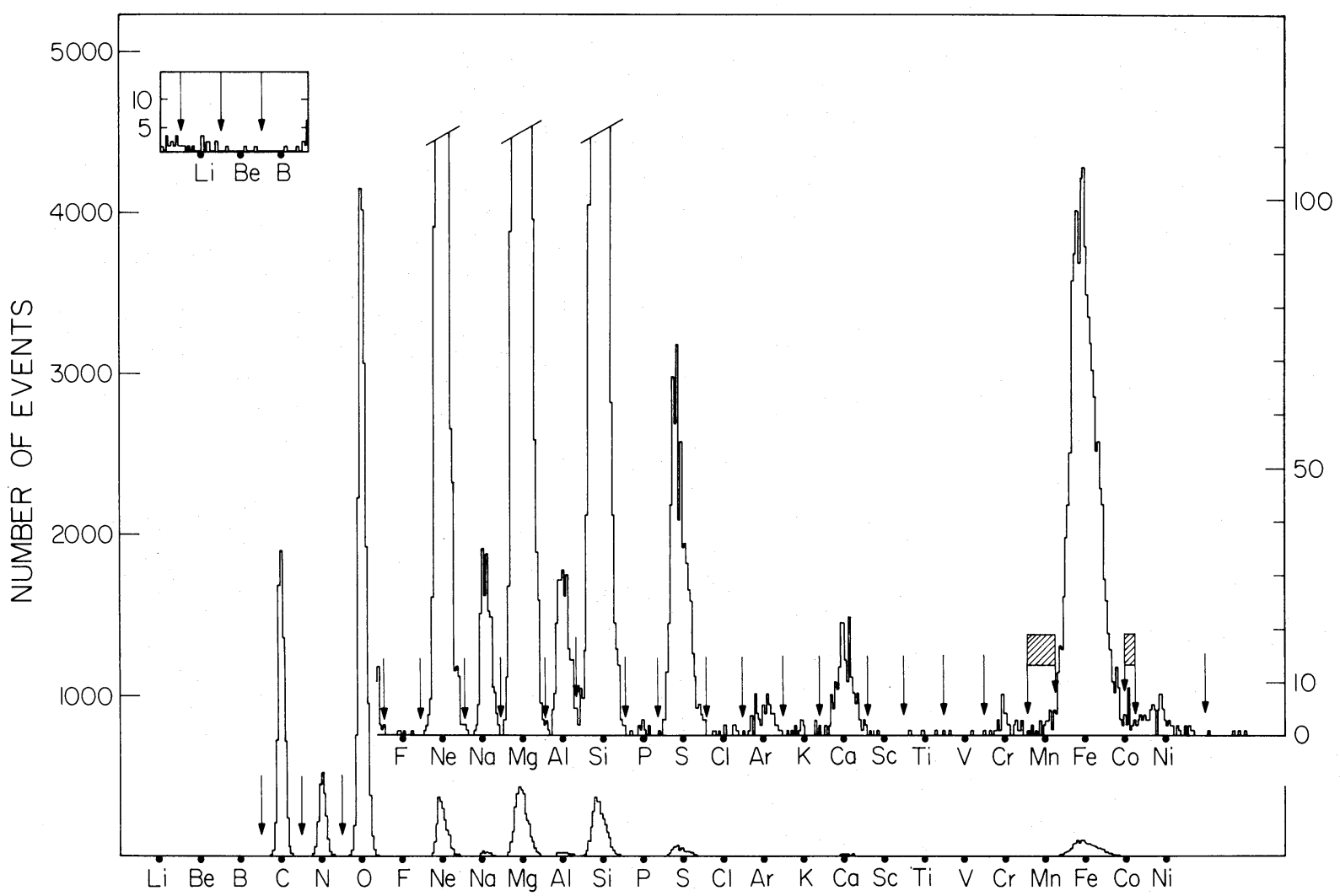

Fig. 2.-A histogram of the average charge measurement $(Z 1+Z 2) / 2$ for events with consistent $Z 1$ and $Z 2$ charge measurements and incident energies in the range $8.7-15 \mathrm{MeV}$ per nucleon. The histogram includes all the events (from LETs on both Voyager spacecraft) used to obtain the relative abundance measurements listed in Tables 1 and 2 for the elements Li through Ni. The abundances were determined by sorting the events into element bins with boundaries indicated by the arrows. Due to spillover from the $\mathrm{Fe}$ peak, $\mathrm{Mn}$ and Co abundances were not obtained, and the shaded regions of the charge scale were excluded from the analysis. Data above oxygen and below carbon are replotted with an expanded vertical scale.

ment is used to improve charge resolution and suppress background, as will be discussed in detail later.

The response of a typical LET is illustrated in Figure 1. The "tracks" of the relatively abundant elements $\mathrm{C}, \mathrm{N}, \mathrm{O}, \mathrm{Ne}$, $\mathrm{Mg}, \mathrm{Si}, \mathrm{S}$, and $\mathrm{Fe}$ are apparent, as are the less populated tracks of $\mathrm{Na}, \mathrm{Al}, \mathrm{Ar}$, and $\mathrm{Ca}$. The finite width of the tracks (and hence the instrumental charge resolution) is determined by the variation of particle incident angles and detector thickness nonuniformities, both of which contribute to variation in the particle path lengths through detector L1 (and L2 for "three-detector events").

For each analyzed event a charge measurement $Z 1$ was calculated by numerically solving for $Z 1$ in the following equation:

$$
T=R\left(\Delta E+E^{\prime}, Z 1, M\right)-R\left(E^{\prime}, Z 1, M\right),
$$

where $T$ is the particle path length in the appropriate L1 detector, averaged over an isotropic particle flux. The mean path length $T$ was measured for each detector using alphaparticles in preflight calibrations. The quantity $\Delta E$ is the particle energy deposition measured in the L1 detector; $E^{\prime}$ is the sum of the energy depositions measured in the L2 and L3 detectors. The quantity $R(E, Z, M)$ is the range in silicon of a nucleus with energy $E$, nuclear charge $Z$, and mass $M$;
$R$ was approximated by a semiempirical expression similar to that developed by Heckman et al. (1960); see Cook (1981). Note that the above equation contains two unknowns, the charge $Z 1$ and the mass $M$. To eliminate the mass as an unknown and permit the solution for the charge $Z 1$, we approximated the nuclear mass as a continuous function of the charge: $M=2(Z 1)$ for $Z 1<20$, and $M=2.132(Z 1)$ for $Z 1>21$, with linear interpolation between $Z 1=20$ and 21 . The small errors incurred for the rarer isotopes produced only small and predictable shifts in the calculated charge measurement.

For three-detector events an additional charge measurement $Z 2$ was calculated by taking the L2 energy measurement as $\Delta E$, the L3 energy measurement as $E^{\prime}$, and $T$ equal to the mean path length through the appropriate L2 detector. The redundant $Z 1$ and $Z 2$ charge measurements were checked for consistency to reduce background. The eliminated events were due primarily to nuclei incident near the edges of either detector L1 or L2. The number of the rejected events and the distribution of their $Z 1$ and $Z 2$ charge measurements indicated that the area of the "edge" of the L1 or L2 detector was roughly the same for the different nuclear species and was about $6 \%$ of the fully active area.

The quality of the data is shown in Figure 2, a histogram 


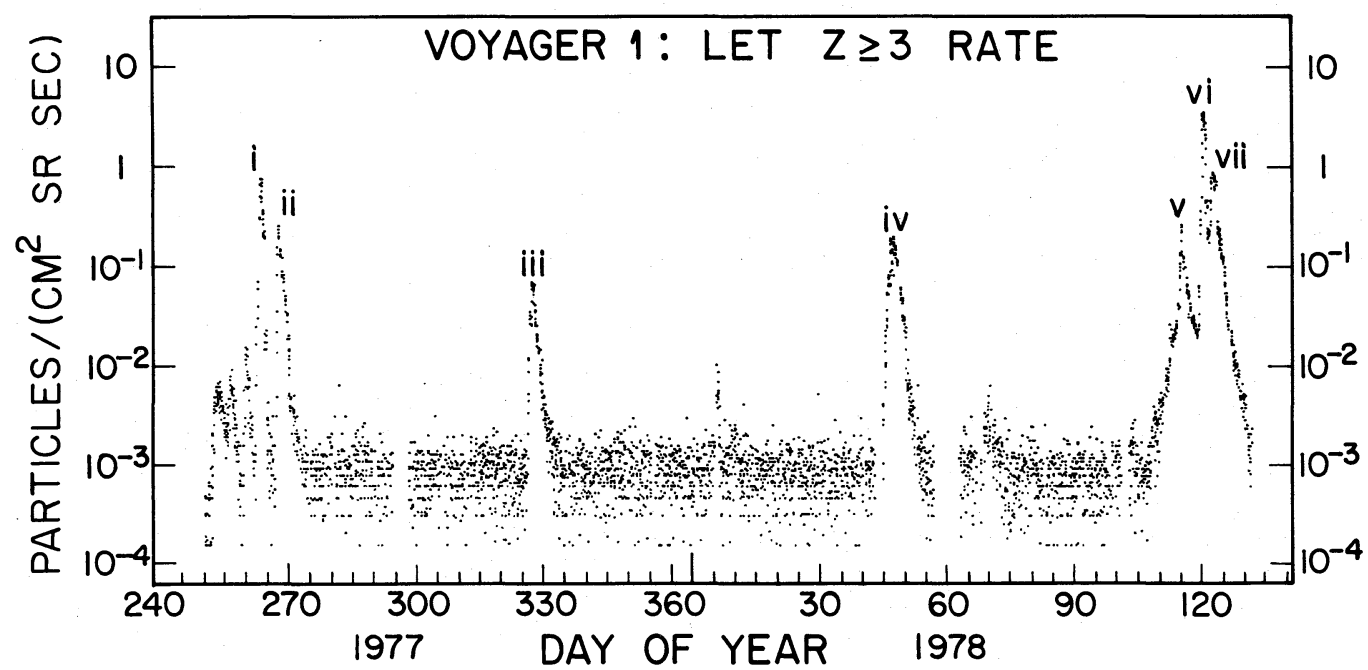

Fig. 3.-The hourly averaged flux of $Z \geq 3$ nuclei measured with the LETs on Voyager 1. The energy threshold for detection varies from about 3 MeV per nucleon for carbon nuclei to $5 \mathrm{MeV}$ per nucleon for iron. The seven largest energetic particle events are labeled i through vii. (The quantization of the flux measurements at low levels corresponds to the detection of a small integer number of nuclei.)

of $(Z 1+Z 2) / 2$ for charge-consistent events with energies from 8.7 to $15 \mathrm{MeV}$ per nucleon. The histogram includes all the events from LETs on both Voyager spacecraft which were used to obtain the abundances of elements from $\mathrm{Li}$ through $\mathrm{Ni}$ presented in Tables 1 and 2 . The rms charge resolution ranges from 0.08 units at carbon to 0.27 units at iron.

\section{OBSERVATIONS}

The seven largest energetic particle events (labeled i through vii in Fig. 3) which occurred in the 1977 September through 1978 May period were selected for study because of the statistical accuracy available for abundance measurements. Voyager 1 and Voyager 2 were within $3 \mathrm{AU}$ of the Sun during this time. The first four events (i through iv) were clearly associated with separate large solar flares (Coffey 1977-1978). The last three events were not well separated but were part of a general particle intensity increase accompanied by several large flares. A compilation of references to energetic particle observations of the first four events may be found in Wibberenz (1979).

We present two different sets of observational results for each flare event: (1) abundance ratio measurements for the nuclei He through Ni listed in Tables 1 and 2, and (2) fluence (time-integrated flux) measurements for some of the more abundant nuclei (namely, C, O, Ne, Mg, Si, and $\mathrm{Fe}+\mathrm{Ni}$ ), listed in Table 3. In both cases we have combined the data from Voyager 1 and Voyager 2 in order to improve statistical accuracy. Except where noted, the results presented in Tables 1 and 2 are identical to those given earlier in Cook, Stone, and Vogt (1980) and are reproduced here for completeness.

We have found our SEP abundance measurements to be rather insensitive to the choice of averaging time period and averaging technique since most elemental ratios remain

TABLE 1

Abundance Results for He and Abundance UpPer Limits for Li, Be, and B

\begin{tabular}{|c|c|c|c|c|c|c|c|}
\hline \multirow{2}{*}{$\begin{array}{l}\text { ELEMENT RATIO } \\
\text { AND } \\
\text { ENERGY RANGE }\end{array}$} & \multicolumn{7}{|c|}{ Solar Energetic Particle Event ${ }^{a}$} \\
\hline & $\mathrm{i}$ & ii & iii & iv & $\mathrm{v}$ & vi & vii \\
\hline $\begin{array}{l}\text { 4.6-7.8 } \mathrm{MeV} \text { per nucleon: } \\
\mathrm{He} / \mathrm{Si} \ldots \ldots \ldots \ldots \ldots \ldots \ldots\end{array}$ & $468 \pm 60^{b}$ & $433 \pm 45$ & $565 \pm 49$ & $384 \pm 15$ & $386 \pm 19$ & $97^{\mathrm{c}}$ & $240 \pm 6$ \\
\hline $\begin{array}{l}5.9-9.3 \mathrm{MeV} \text { per nucleon: } \\
\mathrm{Li} / \mathrm{O} \ldots \ldots \ldots \ldots \ldots \ldots \ldots \ldots \\
\mathrm{Be} / \mathrm{O} \ldots \ldots \ldots \ldots \ldots \ldots \ldots \ldots \\
\mathrm{B} / \mathrm{O} \ldots \ldots \ldots \ldots \ldots \ldots \ldots\end{array}$ & $\begin{array}{l}<0.006^{\mathrm{d}} \\
<0.0024 \\
<0.0024\end{array}$ & $\begin{array}{l}<0.007 \\
<0.005 \\
<0.003\end{array}$ & $\begin{array}{l}<0.003 \\
<0.003 \\
<0.003\end{array}$ & $\begin{array}{l}<0.0011 \\
<0.0006 \\
<0.0006\end{array}$ & $\begin{array}{l}<0.0008 \\
<0.0019 \\
<0.0014\end{array}$ & $\begin{array}{l}<0.0050 \\
<0.0005 \\
<0.0004\end{array}$ & $\begin{array}{l}<0.0016 \\
<0.0004 \\
<0.0004\end{array}$ \\
\hline
\end{tabular}

a The events correspond to the time periods listed in Table 2.

${ }^{\mathrm{b}}$ The $( \pm 1 \sigma)$ uncertainties include the effect of counting statistics only.

${ }^{c}$ Result differs from that given in Cook, Stone, and Vogt 1980 because of the consideration of high rate effects in the instrument. The possible error due to high rate effects exceeds the statistical error (see Cook 1981).

d $84 \%$ confidence upper limits. 
TABLE 2

Numbers of Nuclei Detected and Abundances Relative to Silicon, $8.7-15 \mathrm{MeV}$ per Nucleon

\begin{tabular}{|c|c|c|c|c|c|c|c|c|c|c|c|c|c|c|c|}
\hline \multirow{2}{*}{$\frac{Z}{6 \ldots \ldots \ldots}$} & \multirow{2}{*}{$\frac{\text { Element }}{\mathrm{C}}$} & \multicolumn{2}{|c|}{$\begin{array}{c}1977 \\
\text { Sep } 200000 \mathrm{UT} \\
\text { to } \\
\text { Sep } 220000 \mathrm{UT}\end{array}$} & \multicolumn{2}{|c|}{$\begin{array}{c}1977 \\
\text { Sep } 250000 \text { UT } \\
\text { to } \\
\text { Sep } 271200 \text { UT }\end{array}$} & \multicolumn{2}{|c|}{$\begin{array}{c}1977 \\
\text { Nov } 230600 \mathrm{UT} \\
\text { to } \\
\text { Nov } 261200 \mathrm{UT}\end{array}$} & \multicolumn{2}{|c|}{$\begin{array}{c}1978 \\
\text { Feb } 150000 \mathrm{UT} \\
\text { to } \\
\text { Feb } 180600 \mathrm{UT}\end{array}$} & \multicolumn{2}{|c|}{$\begin{array}{c}1978 \\
\text { Apr } 241800 \mathrm{UT} \\
\text { to } \\
\text { Apr } 281200 \mathrm{UT}\end{array}$} & \multicolumn{2}{|c|}{$\begin{array}{c}1978 \\
\text { Apr } 290600 \mathrm{UT} \\
\text { to } \\
\text { May } 10000 \mathrm{UT}\end{array}$} & \multicolumn{2}{|c|}{ 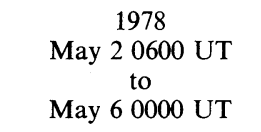 } \\
\hline & & 151 & $3.02 \pm 0.49^{\mathrm{a}}$ & 134 & $1.20 \pm 0.15$ & 72 & $2.12 \pm 0.44$ & 519 & $3.58 \pm 0.34$ & 658 & $4.39 \pm 0.40$ & 3727 & $1.96 \pm 0.06$ & 1765 & $3.90 \pm 0.20$ \\
\hline $7 \ldots \ldots \ldots$ & $\mathrm{N}$ & 35 & $0.70 \pm 0.15$ & 42 & $0.37 \pm 0.07$ & 22 & $0.65 \pm 0.18$ & 133 & $0.92 \pm 0.11$ & 152 & $1.01 \pm 0.12$ & 1230 & $0.65 \pm 0.02$ & 482 & $1.06 \pm 0.07$ \\
\hline $8 \ldots \ldots \ldots$ & $\mathrm{O}$ & 285 & $5.70 \pm 0.87$ & 347 & $3.10 \pm 0.34$ & 179 & $5.26 \pm 0.98$ & 1113 & $7.68 \pm 0.68$ & 1252 & $8.35 \pm 0.72$ & 10956 & $5.77 \pm 0.14$ & 3797 & $8.38 \pm 0.42$ \\
\hline$\cdots$ & $\mathrm{F}$ & 0 & $<0.04$ & 0 & $<0.02$ & 0 & $<0.05$ & 0 & $<0.013$ & 0 & $<0.012$ & 3 & $<0.003^{\mathrm{b}}$ & 1 & $<0.007^{\mathrm{b}}$ \\
\hline ...... & $\mathrm{Ne}$ & 49 & $0.98 \pm 0.20$ & 87 & $0.78 \pm 0.11$ & 30 & $0.88 \pm 0.22$ & 162 & $1.12 \pm 0.13$ & 156 & $1.04 \pm 0.12$ & 1153 & $0.61 \pm 0.02$ & 413 & $0.91 \pm 0.06$ \\
\hline 11. & $\mathrm{Na}$ & 2 & $0.040_{-0.026}^{+0.053}$ & 10 & $0.09_{-0.03}^{+0.04}$ & 2 & $0.06_{-0.04}^{+0.08}$ & 13 & $0.090_{-0.025}^{+0.032}$ & 13 & $0.087_{-0.024}^{+0.031}$ & 112 & $0.059 \pm 0.006$ & 43 & $0.095 \pm 0.015$ \\
\hline 12 . & $\mathrm{Mg}$ & 49 & $0.98 \pm 0.20$ & 97 & $0.87 \pm 0.12$ & 30 & $0.88 \pm 0.22$ & 236 & $1.63 \pm 0.17$ & 222 & $1.48 \pm 0.16$ & 1956 & $1.03 \pm 0.03$ & 709 & $1.57 \pm 0.09$ \\
\hline 13. & $\mathrm{Al}$ & 5 & $0.10_{-0.05}^{+0.07}$ & 12 & $0.11_{-0.03}^{+0.04}$ & 4 & $0.12_{-0.06}^{+0.09}$ & 9 & $0.06_{-0.02}^{+0.03}$ & 21 & $0.14 \pm 0.03$ & 139 & $0.073 \pm 0.006$ & 47 & $0.104 \pm 0.016$ \\
\hline 14 & $\mathrm{Si}$ & 50 & $\equiv 1.0$ & 112 & $\equiv 1.0$ & 34 & $\equiv 1.0$ & 145 & $\equiv 1.0$ & 150 & $\equiv 1.0$ & 1898 & $\equiv 1.0$ & 453 & $\equiv 1.0$ \\
\hline 15 & $\mathbf{P}$ & 0 & $<0.04$ & 1 & $<0.03^{b}$ & 0 & $<0.05$ & 1 & $<0.023^{b}$ & 1 & $<0.022^{\mathrm{b}}$ & 7 & $<0.006^{\mathrm{b}}$ & 0 & $<0.004$ \\
\hline 16 & $\mathrm{~S}$ & 6 & $0.12_{-0.05}^{+0.07}$ & 27 & $0.24 \pm 0.05$ & 14 & $0.41_{-0.11}^{+0.14}$ & 31 & $0.21 \pm 0.04$ & 36 & $0.24 \pm 0.05$ & 401 & $0.21 \pm 0.01$ & 63 & $0.14 \pm 0.02$ \\
\hline 17 & $\mathrm{Cl}$ & 2 & $<0.09^{\mathrm{b}}$ & 0 & $<0.02$ & 0 & $<0.05$ & 0 & $<0.013$ & 2 & $<0.031^{\mathrm{b}}$ & 6 & $<0.005^{\mathrm{b}}$ & 0 & $<0.004$ \\
\hline $18 \ldots \ldots \ldots$ & $\mathrm{Ar}$ & 3 & $0.06_{-0.03}^{+0.06}$ & 6 & $0.054_{-0.021}^{+0.032}$ & 2 & $0.06_{-0.04}^{+0.08}$ & 6 & $0.041_{-0.017}^{+0.025}$ & 1 & $0.007_{-0.006}^{+0.015}$ & 37 & $0.019 \pm 0.003$ & 6 & $0.013_{-0.005}^{+0.008}$ \\
\hline $19 \ldots \ldots \ldots$ & $\mathrm{K}$ & 0 & $<0.04^{-0.03}$ & 1 & $<0.03^{\mathrm{b}}$ & 0 & $<0.05$ & 1 & $<0.023^{\mathrm{b}}$ & 0 & $<0.012$ & 10 & $<0.008^{\mathrm{b}}$ & 1 & $<0.007^{\mathrm{b}}$ \\
\hline 20 & $\mathrm{Ca}^{\mathrm{c}}$ & 7 & $0.14_{-0.05}^{+0.08}$ & 25 & $0.22 \pm 0.05$ & 4 & $0.12_{-0.06}^{+0.09}$ & 13 & $0.090_{-0.025}^{+0.032}$ & 10 & $0.067_{-0.021}^{+0.029}$ & 106 & $0.056 \pm 0.006$ & 16 & $0.035_{-0.009}^{+0.011}$ \\
\hline 21 & $\mathrm{Sc}$ & 0 & $<0.04$ & 1 & $<0.03^{\mathrm{b}}$ & 0 & $<0.05$ & 0 & $<0.013$ & 0 & $<0.012$ & 2 & $<0.002^{\mathrm{b}}$ & 0 & $<0.004$ \\
\hline 22 & $\mathrm{Ti}$ & 0 & $<0.04$ & 0 & $<0.02$ & 0 & $<0.05$ & 0 & $<0.013$ & 0 & $<0.012$ & 4 & $<0.004^{\mathrm{b}}$ & 0 & $<0.004$ \\
\hline 23 & V & 1 & $<0.07^{\mathrm{b}}$ & 0 & $<0.02$ & 0 & $<0.05$ & 0 & $<0.013$ & 0 & $<0.012$ & 2 & $<0.002^{\mathrm{b}}$ & 0 & $<0.004$ \\
\hline 24 . & $\mathrm{Cr}$ & 0 & $<0.04$ & 2 & $0.018_{-0.012}^{+0.024}$ & 1 & $0.029_{-0.025}^{+0.068}$ & 4 & $0.028_{-0.013}^{+0.022}$ & 3 & $0.020_{-0.011}^{+0.020}$ & 21 & $0.011 \pm 0.002$ & 7 & $0.015_{-0.006}^{+0.008}$ \\
\hline & $\mathrm{Fe}^{\mathrm{c}}$ & 82 & $1.64 \pm 0.29$ & 233 & $2.08 \pm 0.24$ & 53 & & 121. & $0.83 \pm 0.10$ & 90 & $0.60 \pm 0.08$ & 1073 & $0.57 \pm 0.02$ & 170 & $0.38 \pm 0.03$ \\
\hline $28 \ldots \ldots \ldots$ & $\mathrm{Ni}$ & 6 & $0.12_{-0.05}^{+0.07}$ & 15 & $0.13_{-0.03}^{+0.04}$ & 2 & $0.06_{-0.04}^{+0.08}$ & 4 & $0.028_{-0.013}^{+0.022}$ & 5 & $0.033_{-0.015}^{+0.023}$ & 45 & $0.024 \pm 0.004$ & 5 & $0.011_{-0.005}^{+0.008}$ \\
\hline
\end{tabular}

${ }^{\mathrm{a}}$ The $( \pm 1 \sigma)$ uncertainties include the effect of counting statistics only.

$84 \%$ confidence upper limits only are quoted for these elements since possible background contributions have not been assessed.

c Results differ slightly from those given in Cook, Stone, and Vogt 1980 because of the correction of an analysis error in the case of Ca and because of improved analysis technique in the case of Fe. 
TABLE 3

Fluence Measurements

\begin{tabular}{|c|c|c|c|c|c|c|c|c|}
\hline \multirow[b]{2}{*}{$Z$} & \multirow[b]{2}{*}{ ELEMENT } & \multicolumn{7}{|c|}{ Solar Energetic Particle Event ${ }^{a}$} \\
\hline & & $\mathrm{i}$ & ii & iii & iv & $\mathrm{v}$ & $\mathrm{vi}^{\mathrm{b}}$ & vii $^{b}$ \\
\hline \multicolumn{9}{|c|}{ Spectral Index, ${ }^{\mathrm{c}} \gamma$ (assuming $d F / d E \sim E^{-\gamma}$ ) } \\
\hline 6. & $\mathrm{C}$ & $3.29 \pm 0.17^{\mathrm{d}}$ & $2.57 \pm 0.20$ & $4.36 \pm 0.19$ & $3.40 \pm 0.07$ & $2.92 \pm 0.07$ & $1.11 \pm 0.04$ & $2.93 \pm 0.04$ \\
\hline 8 & $\mathrm{O}$ & $3.67 \pm 0.12$ & $2.20 \pm 0.10$ & $4.08 \pm 0.11$ & $3.46 \pm 0.07$ & $2.84 \pm 0.05$ & $1.27 \pm 0.02$ & $3.21 \pm 0.03$ \\
\hline$\ldots \ldots$ & $\mathrm{Ne}$ & $3.98 \pm 0.30$ & $1.90 \pm 0.22$ & $4.30 \pm 0.32$ & $3.38 \pm 0.13$ & $2.54 \pm 0.16$ & $1.29 \pm 0.07$ & $3.09 \pm 0.08$ \\
\hline $12 \ldots$ & $\mathrm{Mg}$ & $3.81 \pm 0.28$ & $2.15 \pm 0.16$ & $3.84 \pm 0.29$ & $3.34 \pm 0.10$ & $2.69 \pm 0.12$ & $1.27 \pm 0.05$ & $3.13 \pm 0.06$ \\
\hline $14 \ldots$ & $\mathrm{Si}$ & $3.12 \pm 0.28$ & $1.60 \pm 0.20$ & $3.45 \pm 0.24$ & $3.36 \pm 0.12$ & $3.12 \pm 0.14$ & $1.71 \pm 0.05$ & $3.47 \pm 0.07$ \\
\hline $26 \ldots$ & $\mathrm{Fe}^{\mathrm{e}}$ & $2.42 \pm 0.25$ & $1.97 \pm 0.12$ & $2.22 \pm 0.25$ & $3.46 \pm 0.13$ & $2.57 \pm 0.19$ & $3.06 \pm 0.06$ & $4.04 \pm 0.11$ \\
\hline \multicolumn{9}{|c|}{ Measured Fluence ${ }^{\mathrm{c}}$ at $7.5 \mathrm{MeV}$ per nucleon, $A\left(\mathrm{~cm}^{2} \text { sr } \mathrm{MeV} \text { per nucleon }\right)^{-1}$} \\
\hline 6 . & $\mathrm{C}$ & $233 \pm 11^{\mathrm{d}}$ & $121 \pm 6$ & $50 \pm 2$ & $323 \pm 7$ & $303 \pm 7$ & $2308 \pm 30$ & $854 \pm 10$ \\
\hline 8 & $\mathrm{O}$ & $521 \pm 17$ & $326 \pm 9$ & $106 \pm 3$ & $773 \pm 13$ & $621 \pm 10$ & $7185 \pm 54$ & $1949 \pm 16$ \\
\hline $10 \ldots$ & $\mathrm{Ne}$ & $80 \pm 6$ & $63 \pm 4$ & $15.2 \pm 1.0$ & $112 \pm 4$ & $81 \pm 4$ & $794 \pm 18$ & $212 \pm 5$ \\
\hline $12 \ldots \ldots$ & $\mathrm{Mg}$ & $99 \pm 7$ & $95 \pm 5$ & $17.1 \pm 1.3$ & $152 \pm 5$ & $106 \pm 4$ & $1318 \pm 23$ & $376 \pm 7$ \\
\hline $14 \ldots \ldots$ & $\mathrm{Si}$ & $80 \pm 6$ & $90 \pm 5$ & $14.3 \pm 1.2$ & $106 \pm 4$ & $75 \pm 3$ & $1529 \pm 25$ & $281 \pm 6$ \\
\hline 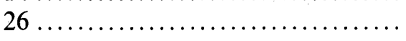 & $\mathrm{Fe}^{\mathrm{e}}$ & $103 \pm 7$ & $252 \pm 9$ & $18.1 \pm 1.2$ & $93 \pm 3$ & $49 \pm 2$ & $1577 \pm 26$ & $141 \pm 4$ \\
\hline Correction factor ${ }^{f}$ & & 1.35 & 1.34 & 1.05 & 1.11 & 1.14 & 1.005 & 1.20 \\
\hline
\end{tabular}

a Observation periods: i, 1977 Sep 191200 UT to 1977 Sep 221500 UT; ii, 1977 Sep 240600 UT to 1977 Sep 271500 UT; iii, 1977 Nov 221200 UT to 1977 Nov 270300 UT; iv, 1978 Feb 132100 UT to 1978 Feb 210900 UT; v, 1978 Apr 240000 UT to 1978 Apr 290300 UT; vi, 1978 Apr 290600 UT to 1978 May 10000 UT; vii, 1978 May 20600 UT to 1978 May 71800 UT.

b Spectra were not well fitted by a power function; “ $\gamma$ " increased with energy (see Cook 1981).

${ }^{c}$ Fluence spectra were obtained by a least squares fit of the form $d F / d E=A(E / 7.5)^{-\gamma}$ to data in six energy bins from 5 to $15 \mathrm{MeV}$ per nucleon.

d $( \pm 1 \sigma)$ statistical uncertainty.

e Includes nickel.

${ }^{\mathrm{f}}$ Estimated multiplicative factor to correct fluences for data gaps which occurred during the observation periods.

relatively constant throughout a given flare event. For example, Figure 4 shows that the $\mathrm{C} / \mathrm{O}, \mathrm{Ne} / \mathrm{O}, \mathrm{Mg} / \mathrm{O}$, and $\mathrm{Si} / \mathrm{O}$ remain nearly constant during the SEP events $\mathrm{i}$ and ii. However, the $\mathrm{Fe} / \mathrm{O}$ ratio did show a systematic decrease during the first $12-24 \mathrm{hr}$ of four out of the seven flare events we studied. For these four events we compare in Table $4 \mathrm{Fe} / \mathrm{O}$ abundance ratios measured with two different averaging time periods and two different averaging techniques. Even for the $\mathrm{Fe} / \mathrm{O}$ ratio it can be seen that in practice the choice of averaging time period and technique causes differences in the measurements which are only as large as the statistical errors and are small compared with the flare-to-flare variations. Thus, for the primary abundance results listed in Table 2 we have chosen to use the simplest averaging technique, in which the relative abundances are formed as ratios of the total numbers of analyzed events obtained for the different elements in the chosen time period. The raw event counts on which such ratios are based are presented in Table 2 such that the reader may form any desired abundance ratio and simply obtain its statistical uncertainty.

The primary abundance results presented in Table 2 for the elements carbon through nickel and the upper limits (Table 1) for the $\mathrm{Li} / \mathrm{O}, \mathrm{Be} / \mathrm{O}$, and $\mathrm{B} / \mathrm{O}$ ratios are based entirely on our highest quality data-three-detector events for which the particle charge is redundantly measured and checked for consistency. All of the results presented in Tables 1 and 2 were averaged over time periods chosen to exclude event onset times where the $\mathrm{Fe} / \mathrm{O}$ ratio was elevated because of propagation effects. Typical averaging periods are shown in Figure 4. The abundance results for carbon through nickel were obtained over a single common velocity interval corresponding to $8.7-15 \mathrm{MeV}$ per nucleon.

Measurements of the fluences for the elements $\mathrm{C}, \mathrm{O}, \mathrm{Ne}, \mathrm{Mg}$, $\mathrm{Si}$, and $\mathrm{Fe}+\mathrm{Ni}$ are summarized in Table 3 by listing the coefficients $A$ and $\gamma$ of a power-law fit $d F / d E=A(E / 7.5)^{-\gamma}$ $\left(\mathrm{cm}^{2} \text { sr MeV per nucleon }\right)^{-1}$ to fluence spectra measured for each element in the same six energy bins covering the interval 5-15 MeV per nucleon. The fluence measurements were obtained for entire flare events as a convenience to those interested in total integrated spectra.

The abundance results of Tables 1 and 2 have been compared with other reported observations-McGuire, von Rosenvinge, and McDonald (1979) for flare events i, ii, iii, and iv, and Dietrich and Simpson (1978) for event ii. For most of the elements (namely $\mathrm{He}, \mathrm{C}, \mathrm{N}, \mathrm{O}, \mathrm{Ne}, \mathrm{Na}$, $\mathrm{Mg}, \mathrm{Al}, \mathrm{Si}, \mathrm{S}, \mathrm{Ar}, \mathrm{Ca}$, and $\mathrm{Fe}$ ), the various measurements generally agree within statistical errors after being corrected to a common energy interval. However, our results do not support the high abundances of the rare elements $\mathrm{B}, \mathrm{F}$, and $\mathrm{Cr}$ reported by Dietrich and Simpson (1978) for event ii. Their finite results are significantly higher than our upper limits for $B$ and $F$ and our finite value for $C r$. Since the B, F, and $\mathrm{Cr}$ results of Dietrich and Simpson (1978) are based on only a few analyzed events, there is concern about possible contamination from the more abundant nearby elements $\mathrm{C}, \mathrm{O}, \mathrm{Ne}$, and $\mathrm{Fe}$ (see Mewaldt 1980). 


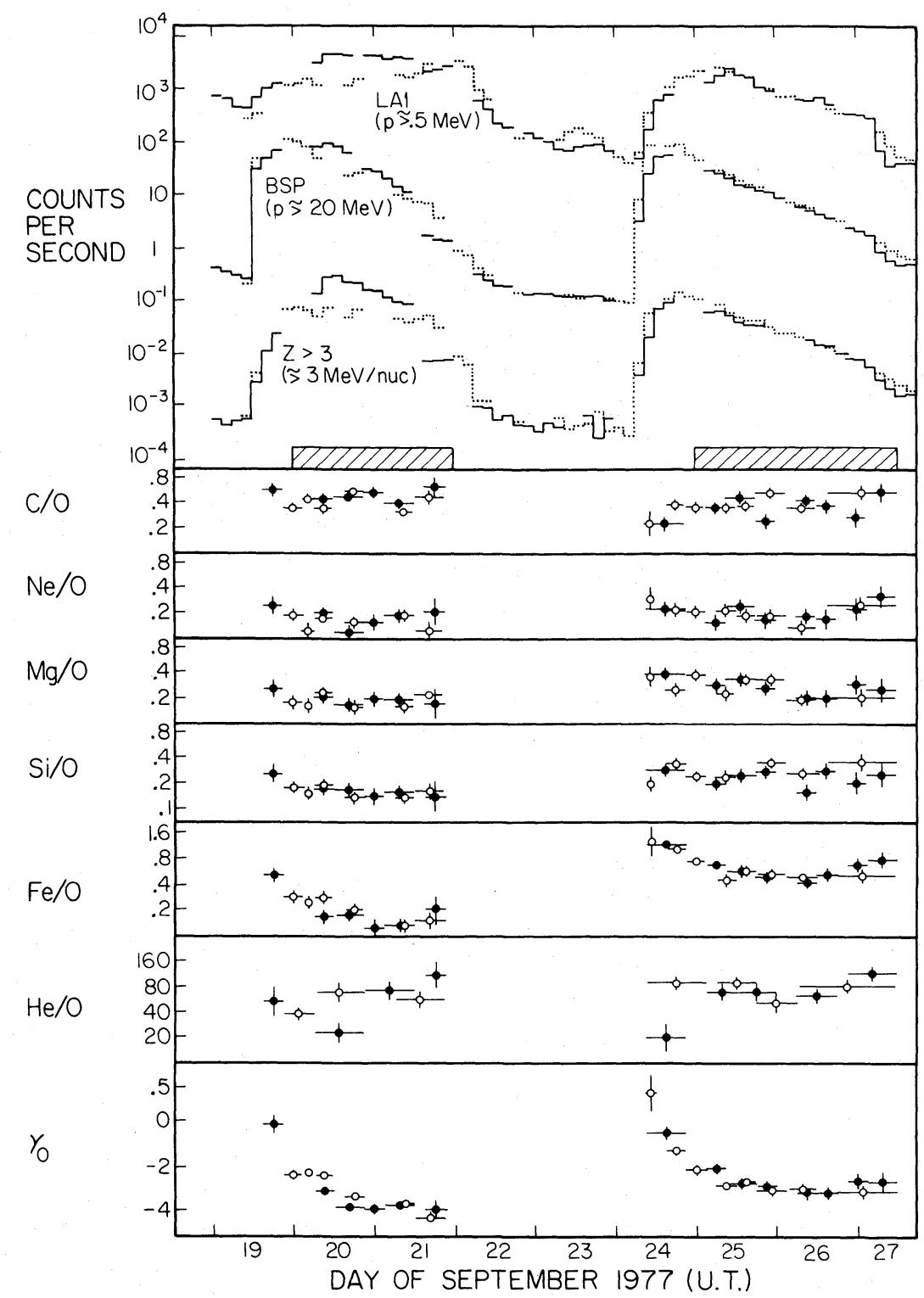

FIG. 4.-Energetic particle event time profiles. Various count rates, element ratios, and the oxygen spectral index are plotted separately for Voyager 1 (solid line and filled circles) and Voyager 2 (dotted line and open circles). The count rates LA1, BSP, and $Z \geq 3$ correspond, respectively, to protons above $0.5 \mathrm{MeV}$, protons above $20 \mathrm{MeV}$, and $Z \geq 3$ above an energy threshold which varies from about $3 \mathrm{MeV}$ per nucleon for carbon to $5 \mathrm{MeV}$ per nucleon for iron. The element ratios are for the common energy per nucleon interval 5-15 MeV per nucleon, except in the case of the $\mathrm{He} / \mathrm{O}$ ratio, where the energy range is 4.0-7.8 MeV per nucleon. The oxygen spectral index was obtained from the ratio of oxygen events in the 4.0-6.1 and 6.1-15 MeV per nucleon energy intervals, assuming $d J / d E \propto E^{-\gamma}$. The crosshatched boxes illustrate the time interval selection for the abundance measurements presented in Tables 1 and 2 .

\section{DISCUSSION}

An overview of our SEP abundance results and their relationship to other solar abundance measurements is shown in Figure 5, a plot of the SEP abundance measurements (from Tables 1 and 2) for all seven flare events, along with abundances for the photosphere, corona, and solar wind. The SEP composition is seen to vary from flare to flare. However, the average SEP abundances (normalized to silicon) are similar to abundances from the other sources for all the elements shown except $\mathrm{C}, \mathrm{N}$, and $\mathrm{O}$, where the SEP values are persistently low relative to the photosphere. The agree- ment between SEP and coronal composition seen in Figure 5 is improved over our earlier comparison (Cook, Stone, and Vogt 1980) as a result of the use of the most recent coronal abundances from the X-ray measurements of Veck and Parkinson (1981).

For the purpose of studying solar composition, we wish to concentrate on those flare events for which the measured SEP abundance ratios have the least dependence on energy per nucleon (and thus possible acceleration and propagation effects) and, therefore, might have values which equal those in the pre-accelerated plasma at the SEP acceleration site. 
TABLE 4

Fe/O Abundance Ratio (8.7-15 MeV per Nucleon)

\begin{tabular}{|c|c|c|c|c|}
\hline \multirow{2}{*}{$\begin{array}{c}\text { ABUNDANCE } \\
\text { AVERAGING TIME } \\
\text { INTERVAL AND } \\
\text { AVERAGING TECHNIQUE }\end{array}$} & \multicolumn{4}{|c|}{ Solar Energetic Particle Event } \\
\hline & $\begin{array}{c}1977 \\
\text { Sep } 19\end{array}$ & $\begin{array}{c}1977 \\
\text { Sep } 24\end{array}$ & $\begin{array}{c}1977 \\
\text { Nov } 22\end{array}$ & $\begin{array}{c}1978 \\
\text { Feb } 13\end{array}$ \\
\hline \multicolumn{5}{|l|}{ Full event: } \\
\hline Flux weighted ${ }^{\mathrm{a}}$ & $0.28 \pm 0.05$ & $0.78 \pm 0.06$ & $0.31 \pm 0.04$ & $0.12 \pm 0.01$ \\
\hline PHA event weighted ${ }^{\mathrm{b}}$ & $0.33 \pm 0.04$ & $0.78 \pm 0.05$ & $0.35 \pm 0.04$ & $0.13 \pm 0.01$ \\
\hline \multicolumn{5}{|l|}{ Partial event (onset excluded): } \\
\hline Flux weighted $\ldots \ldots \ldots \ldots \ldots$ & $0.24 \pm 0.04$ & $0.67 \pm 0.07$ & $0.26 \pm 0.05$ & $0.10 \pm 0.01$ \\
\hline PHA event weighted $\ldots \ldots \ldots \ldots \ldots \ldots \ldots$ & $0.29 \pm 0.04$ & $0.67 \pm 0.06$ & $0.30 \pm 0.05$ & $0.11 \pm 0.01$ \\
\hline
\end{tabular}

${ }^{\text {a }}$ Calculated as a ratio of fluences (i.e., time-integrated fluxes).

${ }^{\mathrm{b}}$ Calculated as a simple ratio of event counts.

We have therefore selected four events (i, ii, iv, and v) for further study and have rejected three events (iii, vi, and vii) which, as shown in Figure 6, have large statistically significant differences of the $(\mathrm{Fe}+\mathrm{Ni})$ spectral index from the spectral indices of the elements $\mathrm{C}, \mathrm{O}, \mathrm{Ne}, \mathrm{Mg}$, and $\mathrm{Si}$.

The SEP composition varies even among the selected four flare events. The systematic nature of this flare-to-flare composition variation is shown in Figure 7 by comparing the SEP composition results for each of the four selected flare events to the "four-flare average" composition, formed as the logarithmic average or geometric mean of abundances for the four flares. (The logarithmic average is used to avoid undue weighting of larger abundance values when, as for the $\mathrm{Fe} / \mathrm{Si}$ ratio, the flare-to-flare abundance variation is large.) As noted by Cook, Stone, and Vogt (1980), the correlation shown in Figure 7 suggests that the four-flare average may represent a characteristic solar particle composition about which there is a systematic flare-to-flare variation which is a monotonic function of nuclear charge $Z$ (or a related atomic parameter such as mass). Since the flare-to-flare variation about the average appears to have a simple dependence on $Z$, we are able to compare the four-flare average abundances with those from other solar sources, knowing that the same comparisons, when made separately for the

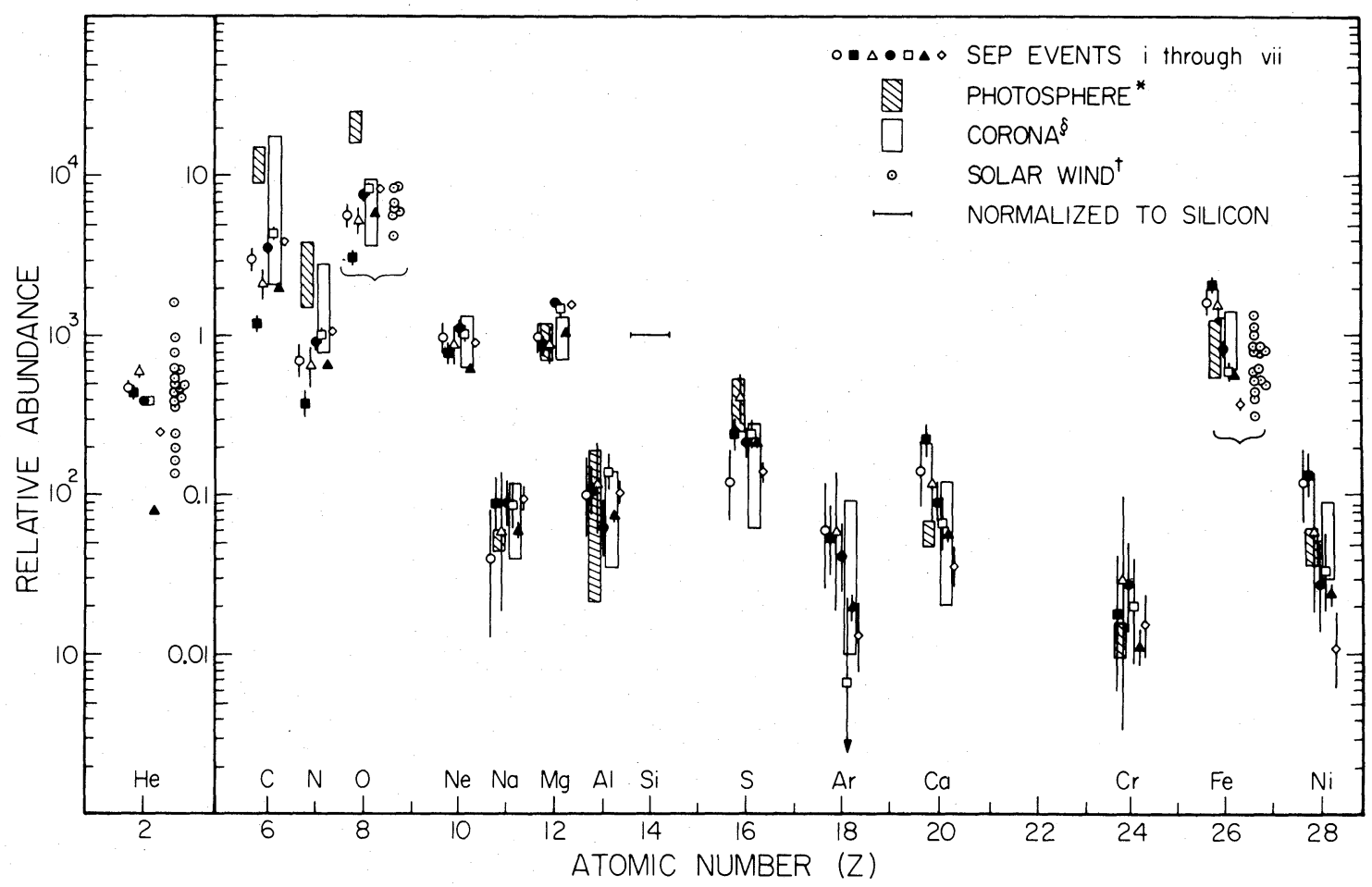

FIG. 5.-An overview of the SEP abundance results in comparison with abundance results for the solar photosphere, corona, and solar wind. All abundances are normalized to silicon. References: $\left({ }^{*}\right)$ Meyer and Reeves (1977), (†) Bame et al. (1975), (§) Veck and Parkinson (1981), except for the elements C, N, and Al, for which abundances were taken from Meyer and Reeves (1977). 


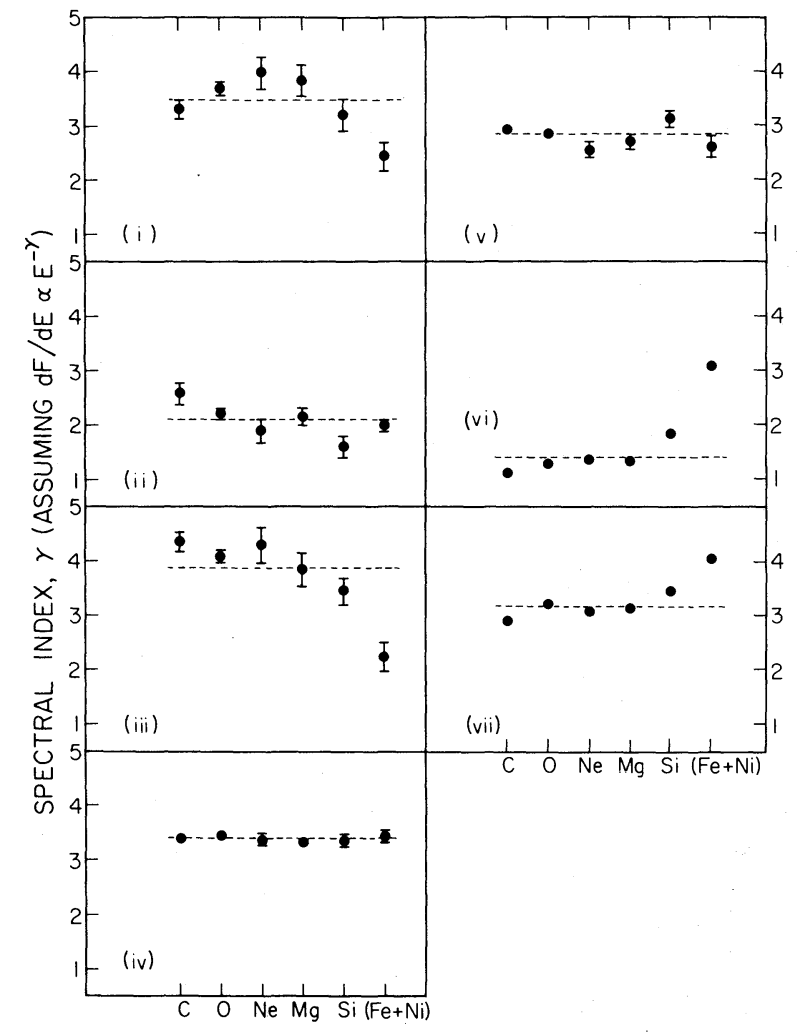

FIG. 6
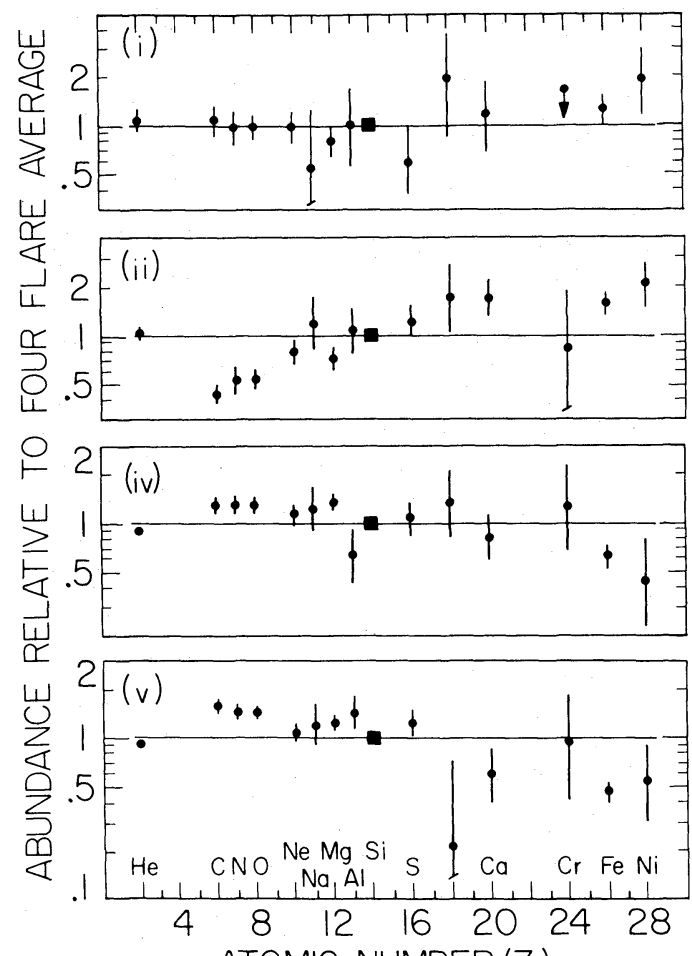

ATOMIC NUMBER (Z)

FIG. 7

Fig. 6.-Spectral indices for the elements $\mathrm{C}, \mathrm{O}, \mathrm{Ne}, \mathrm{Mg}, \mathrm{Si}$, and $\mathrm{Fe}+\mathrm{Ni}$ in flare events $\mathrm{i}$ through vii.

FIG. 7.-Systematics of SEP flare-to-flare composition variations. For each of the flare events $i$, ii, iv, and v, the SEP abundances (from Tables 1 and 2 and normalized to silicon, $\mathbf{0}$ ) were divided by the four-flare average abundances (from Table 5) and plotted vs. nuclear charge.

TABLE 5

Relative Abundances in Solar Energetic Particles and in the Photosphere, Corona, and Solar Wind

\begin{tabular}{|c|c|c|c|c|c|c|}
\hline \multirow[b]{2}{*}{$Z$} & \multirow[b]{2}{*}{ ELEMENT } & \multirow{2}{*}{$\begin{array}{c}\text { SEP FOUR- } \\
\text { FLARE AVERAGE }\end{array}$} & \multirow[b]{2}{*}{ PHOTOSPHERE } & \multicolumn{2}{|c|}{ CORONA $^{\mathrm{c}}$} & \multirow{2}{*}{$\begin{array}{l}\text { SOLAR }^{d} \\
\text { WIND }\end{array}$} \\
\hline & & & & (1) & (2) & \\
\hline 2 . & $\mathrm{He}$ & $416 \pm 19$ & & $\ldots$ & & $530 \pm 250$ \\
\hline …......... & $\mathrm{C}$ & $2.74 \pm 0.17$ & $12 \pm 3$ & $\ldots$ & $5.5(3)$ & $80-20$ \\
\hline ..... & $\mathrm{N}$ & $0.70 \pm 0.06$ & $2.4(1.6)$ & & $1.5(2)$ & \\
\hline $8 \ldots$ & $\mathrm{O}$ & $5.80 \pm 0.34$ & $21 \pm 5$ & $6.3 \pm 2.8$ & $\ldots$ & $6.6 \pm 3.7$ \\
\hline ........ & $\mathrm{Ne}$ & $0.97 \pm 0.07$ & & $1.0 \pm 0.3$ & $\ldots$ & $1.0 \pm 0.5$ \\
\hline $11 \ldots \ldots \ldots \ldots \ldots \ldots$ & $\mathrm{Na}$ & $0.07(1.4)^{\mathrm{e}}$ & $0.052 \pm 0.008$ & $0.08 \pm 0.04$ & $\ldots$ & . \\
\hline $12 \ldots \ldots \ldots \ldots \ldots \ldots$ & $\mathrm{Mg}$ & $1.20 \pm 0.09$ & $0.93 \pm 0.25$ & $1.0 \pm 0.3$ & & $\ldots$ \\
\hline ........ & $\mathrm{Al}$ & $0.10 \pm 0.02$ & $0.063(3)$ & $\ldots$ & $0.07(2)$ & $\ldots$ \\
\hline $14 \ldots \ldots \ldots \ldots \ldots \ldots$ & $\mathrm{Si}$ & $\equiv 1$ & $\equiv 1$ & $\equiv 1$ & $\equiv 1$ & $\equiv 1$ \\
\hline $16 \ldots \ldots \ldots \ldots \ldots \ldots$ & $\mathrm{S}$ & $0.20 \pm 0.04$ & $0.39 \pm 0.14$ & $0.17 \pm 0.11$ & $\ldots$ & $\ldots$ \\
\hline $18 \ldots \ldots \ldots \ldots \ldots \ldots$ & $\mathrm{Ar}$ & $0.03(1.9)$ & & $0.05 \pm 0.04$ & $\begin{array}{l}\cdots \\
\cdots\end{array}$ & $0.024 \pm 0.013$ \\
\hline $20 \ldots \ldots \ldots \ldots \ldots \ldots$ & $\mathrm{Ca}$ & $0.12 \pm 0.02$ & $0.057 \pm 0.010$ & $0.07 \pm 0.05$ & $\ldots$ & $\ldots$ \\
\hline $24 \ldots \ldots \ldots \ldots \ldots \ldots$ & $\mathrm{Cr}$ & $0.02(1.8)$ & $0.0125 \pm 0.0030$ & & $\ldots$ & 然 \\
\hline $26 \ldots \ldots \ldots \ldots \ldots \ldots \ldots$ & $\mathrm{Fe}$ & $1.14 \pm 0.08$ & $0.89 \pm 0.35$ & $1.0 \pm 0.4$ & $\begin{array}{l}\cdots \\
\cdots\end{array}$ & $0.7 \pm 0.5$ \\
\hline $28 \ldots \ldots \ldots \ldots \ldots \ldots$ & $\mathrm{Ni}$ & $0.06(1.3)$ & $0.048 \pm 0.012$ & $0.06 \pm 0.03$ & $\ldots$ & $\ldots$ \\
\hline
\end{tabular}

${ }^{a}$ The logarithmic average of the SEP abundances from flare events i, ii, iv, and $\mathrm{v}$. The $( \pm 1 \sigma)$ uncertainties include the effect of particle counting statistics but not that of flare-to-flare abundance variations.

${ }^{\mathrm{b}}$ Meyer and Reeves 1977.

c (1) Estimated from Parkinson 1977 and Veck and Parkinson 1981, (2) Meyer and Reeves 1977.

d Solar wind abundances relative to silicon were inferred from: $\mathrm{He} / \mathrm{H}=0.04 \pm 0.01, \mathrm{O} / \mathrm{H}=(5 \pm 2) \times 10^{-4}$, $\mathrm{Fe} / \mathrm{H}=(5 \pm 2) \times 10^{-5}, \mathrm{Si} / \mathrm{H}=(7.6 \pm 3) \times 10^{-5}, \mathrm{He} / \mathrm{Ne}=530 \pm 70$, and $\mathrm{Ne} / \mathrm{Ar}=41 \pm 10$ (Boschler and Geiss 1976).

e Numbers in parentheses indicate factors of uncertainty. 

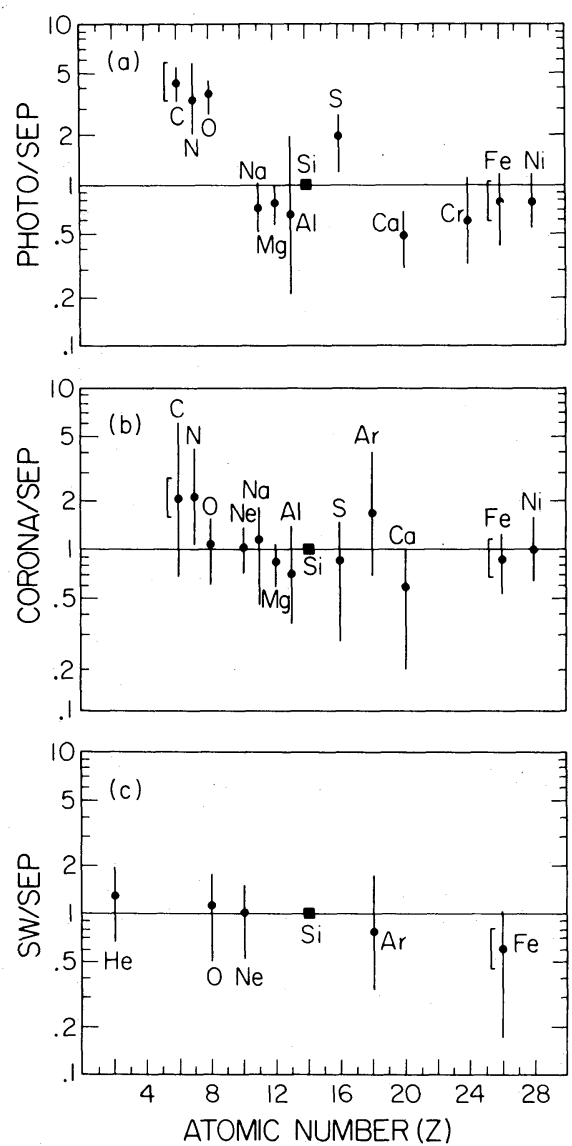

Fig. 8.-Comparison of the four-flare average SEP abundances with abundances from the photosphere, corona, and solar wind. The $( \pm 1 \sigma)$ error bars include the estimated uncertainty in photosphere, corona, or solar wind abundances, and the uncertainty due to particle counting statistics in the average SEP abundances. The $( \pm 1 \sigma)$ uncertainty in the average SEP abundances due to systematic flare-to-flare abundance variations is indicated for carbon and iron by brackets. References: $(a)$ photosphere abundances, Meyer and Reeves (1977); $(b)$ coronal abundances and uncertainties were estimated from Parkinson (1977) and Veck and Parkinson (1981), except for the elements $\mathrm{C}, \mathrm{N}$, and $\mathrm{Al}$, where abundances were taken from Meyer and Reeves (1977); (c) solar wind abundances, Boschler and Geiss (1976).

four-flare events, would differ by small factors which are roughly monotonic functions of $Z$ (for $6 \leq Z \leq 28$ ). Further, the average SEP composition is not very sensitive to our choice of this particular set of four flare events. For example, none of the average abundances for the entire set of seven flare events differ by more than $30 \%$ from the four-flare average abundances. The four-flare average abundances also differ by typically less than $20 \%$ from the "mass-unbiased" baseline SEP abundances compiled by Meyer (1981a) in a study which included our abundance results for all seven flares.

It is interesting that the selection of flare events with SEP abundances that are approximately independent of energy per nucleon does not reduce the range of flare-to-flare variation of the $\mathrm{Fe} / \mathrm{Si}$ ratio, which varies by nearly a factor of 10 in both the full seven-flare set and selected four-flare set. This is consistent with earlier suggestions (Zwickl et al. 1978; Briggs, Armstrong, and Krimigis 1979) that the flare-to-flare variation of SEP abundances may be largely due to variability of the composition of the pre-accelerated plasma at the SEP acceleration sites.

In Figure 8 (also see Table 5) we compare the four-flare average SEP composition with abundances for the photosphere, corona, and solar wind. We have taken the photospheric abundance data from Meyer and Reeves (1977), which is the most recent compilation which covers all the elements of interest here and which also incorporates error estimates. The photospheric abundance data of Meyer and Reeves (1977) are in close agreement with the earlier compilation of Ross and Aller (1976) and with the more recent photospheric abundance determinations by Lambert (1978) and Lambert and Luck (1978) (although Lambert and Luck estimate a significantly smaller uncertainty for the $\mathrm{Al}$ abundance). Figure $8 a$ shows that relative to the photospheric abundance data, the average SEP abundances of $\mathrm{C}, \mathrm{N}, \mathrm{O}$, and to a lesser extent $\mathrm{S}$, are depleted with respect to the other elements in the range $11 \leq Z \leq 28$. The relative depletion of $C, N$, and O in SEPs is now seen to be a persistent effect, as reported in an earlier account of this work (Cook et al. 1979) and by McGuire, von Rosenvinge, and McDonald (1979). The depletion is also present in the earlier data of Teegarden, von Rosenvinge, and McDonald (1973), Crawford et al. (1975), Pellerin (1975), Webber et al. (1975), Nevatia, Durgaprasad, and Biswas (1977), and Mason, Hovestadt, and Gloeckler (1979). The only SEP composition measurements which do not show the depletion are the earliest nuclear emulsion results (e.g., Bertsch, Fichtel, and Reames 1972), which, according to Webber et al. (1975), could be in error as the result of limited charge resolution and detection efficiency.

Figure $8 b$ compares coronal abundances with the average SEP composition. The agreement of the average SEP and coronal abundances is generally within the rather large uncertainties of the coronal measurements, as was also suggested by earlier comparisons with less well determined coronal abundances (see Cook, Stone, and Vogt 1980; Meyer 1981b; Webber 1982). The agreement is particularly notable for $\mathrm{O}$, where both the SEP and coronal abundance are depleted relative to the photosphere. Coronal abundances of $\mathrm{O}$ which are low relative to the photospheric results, but consistent with the SEP values, have been found in both EUV (Withbroe 1975; Flower and Nussbaumer 1975; Mariska 1980) and X-ray studies (Acton, Catura, and Joki 1975; Parkinson 1977).

Figure $8 c$ shows that the solar wind and average SEP elemental abundances are in agreement. Further, Figure 5 shows that the ranges of variation of the $\mathrm{O} / \mathrm{Si}$ and $\mathrm{Fe} / \mathrm{Si}$ ratios are similar in the SEPs and solar wind, although some of the variation of the solar wind measurements may be due to experimental errors (Bame et al. 1975).

There is no evidence for a systematic bias in the average SEP abundances that can account for the large difference between the SEP and photosphere compositions. For example, we consider the SEP $\mathrm{Mg} / \mathrm{O}$ ratio, which is persistently a factor of 4-5 higher than the photosphere value. The SEP $\mathrm{Mg} / \mathrm{O}$ ratio remains relatively constant with time during individual flare events (see, for example, Fig. 4) suggesting that interplanetary propagation has not altered the abundance ratio. Further, as can be seen in Figure 6, the spectral 


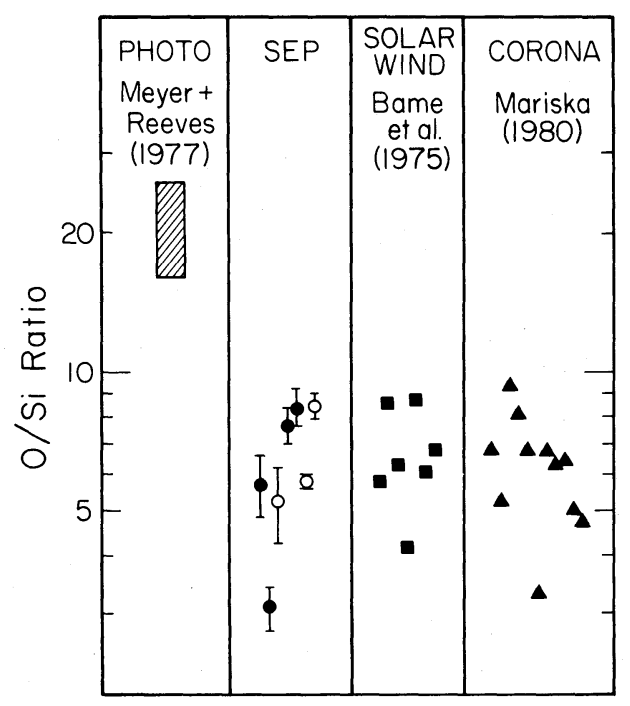

FIG. 9.-Comparison of the $\mathrm{O} / \mathrm{Si}$ ratio measured in the photosphere, SEPs (this work), solar wind, and corona. For the SEP data, the filled circles refer to the four preferred SEP events.

indices of $\mathrm{Mg}$ and $\mathrm{O}$ are nearly the same in each of the seven flares such that the difference of the photosphere and SEP $\mathrm{Mg} / \mathrm{O}$ ratios is not due to systematic differences in the energy per nucleon spectra of $\mathrm{Mg}$ and $\mathrm{O}$ in the 5-15 MeV per nucleon energy range.

To further explore the nature of the difference between the SEP and photosphere compositions, we focus in Figure 9 on the $\mathrm{O} / \mathrm{Si}$ ratio, where measurements are available from all four sources (SEPs, photosphere, solar wind, and corona). The individual coronal measurements (EUV data, Mariska
1980) are for a wide range of different coronal environmentsquiet Sun, coronal hole, active region, and prominence. The $\mathrm{O} / \mathrm{Si}$ ratios measured in SEPs, solar wind, and the corona have a comparable spread and, on the average, are all low relative to the photospheric value by slightly more than a factor of 3. Although the $\mathrm{O} / \mathrm{Si}$ ratios observed in SEPs, solar wind, and the corona could each be biased for independent reasons, the similarity of the depletion of $O$ in SEPs, the solar wind, and the corona suggests that SEPs originate in the corona, and that both the SEPs and the solar wind sample a coronal composition which is persistently different from that measured for the photosphere. The suggestion that the coronal and photospheric elemental compositions are systematically and persistently different has been made independently by Veck and Parkinson (1981) based on their coronal X-ray data.

It is interesting that the $Z$-dependence of the ratios of photospheric abundances to four-flare average SEP abundances (shown in Fig. 8a) is considerably different from the $Z$-dependence of the SEP flare-to-flare abundance variations (shown in Fig. 7). The flare-to-flare SEP abundance variations were fairly smooth monotonic functions of nuclear charge $Z$, for $6 \leq Z \leq 28$, indicating a possible origin in electromagnetic processes during acceleration and propagation. In contrast, the $Z$-dependence of the ratios of photospheric abundances to four-flare average SEP abundances are roughly ordered by a different atomic parameter-the first ionization potential - as noted originally by Hovestadt (1974). In Figure 10, we see that the elements with first ionization potential less than $8 \mathrm{eV}$ form a group in which there is rough agreement between the four-flare average SEP and photospheric abundances. However, the elements $(\mathrm{C}, \mathrm{N}$, and $\mathrm{O})$ with first ionization potential above $11 \mathrm{eV}$ are depleted with respect to the elements with ionization potential below $8 \mathrm{eV}$

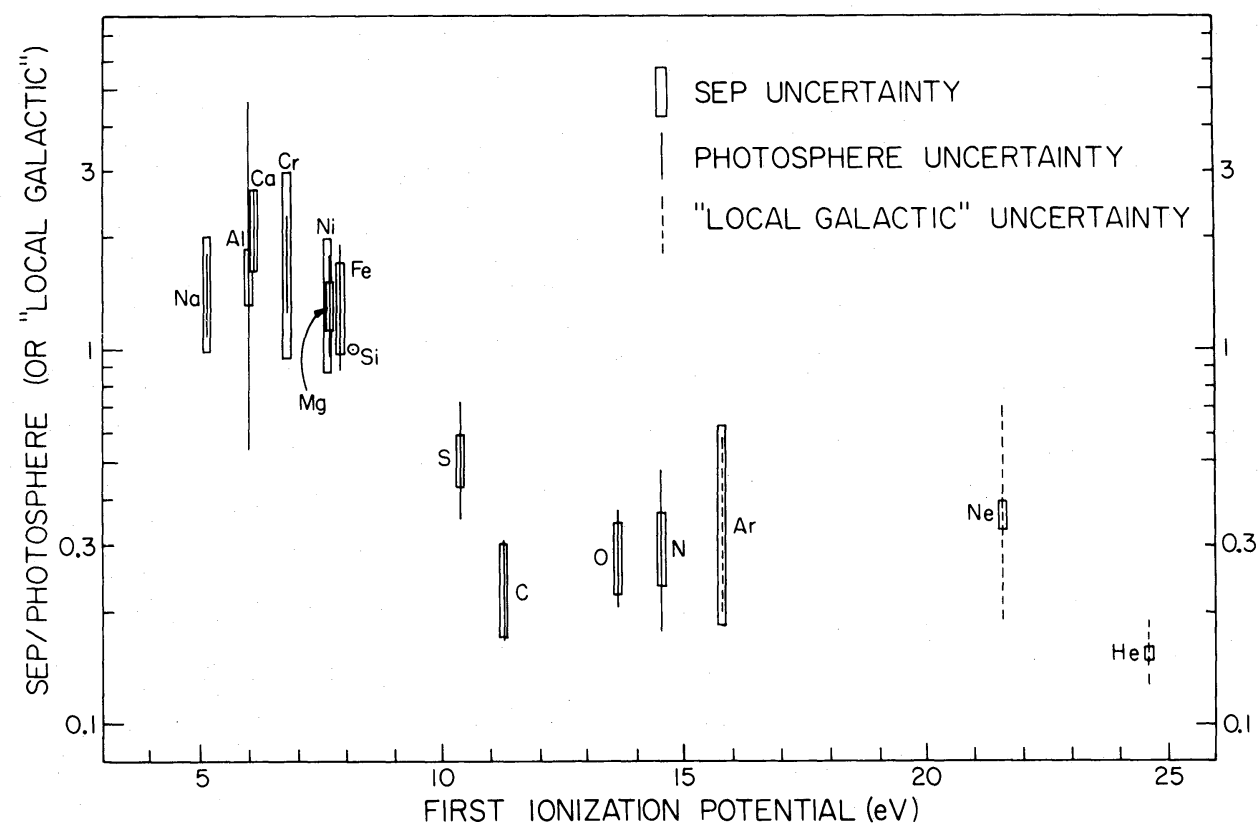

FIG. 10.-Ratios of the four-flare average SEP abundances to photospheric abundances or to "local galactic" abundances (for the elements Ne, Ar, and $\mathrm{He}$ ) plotted vs. first ionization potential. All abundances are normalized to silicon $(\odot)$. The "SEP uncertainty" is the ( $\pm 1 \sigma)$ uncertainty in the four-flare average abundance, including both the effects of particle counting statistics and systematic flare-to-flare abundance variations. 
by a factor of about 5. Sulfur, with an ionization potential near $10 \mathrm{eV}$, appears in the region of transition between the two groups of elements.

The abundances of the other elements ( $\mathrm{Ne}, \mathrm{Ar}$, and $\mathrm{He}$ ) with first ionization potential above $11 \mathrm{eV}$ are not measured in the photosphere. However, in Figure 10 we compare the average SEP abundances of these elements with the best available estimates of their solar abundances from the compilation of "local galactic" abundances by Meyer (1979). The average SEP abundances of $\mathrm{Ne}$, $\mathrm{Ar}$, and $\mathrm{He}$ appear depleted, similar to those of $\mathrm{C}, \mathrm{N}$, and $\mathrm{O}$.

The ordering of SEP to photosphere abundance ratios seen in Figure 10, when taken together with the similarity of SEP, coronal, and solar wind abundances, suggests that first ionization potential or some other related atomic property (such as the cross section for ionization by electron impact) plays an important role in the chemical differentiation of the corona from the photosphere, or, possibly, in measurements of the photospheric abundances (see also Cook 1981;
Meyer $1981 b$ ). The first ionization potential ordering of the SEP to photosphere abundance ratios is qualitatively consistent with the calculations of Geiss (1982), which indicate that ion-neutral separation may occur in the photosphere-corona transition region because of gravitational settling of neutrals.

In conclusion, the results presented here suggest that SEPs originate in the corona, thus providing a measure of the coronal abundances of 15 elements.

We gratefully acknowledge the contributions of the Voyager CRS team, consisting of scientists and engineers at the California Institute of Technology, the Goddard Space Flight Center, the University of Arizona, and the University of New Hampshire. We especially thank Dr. R. A. Mewaldt for his ideas, comments, and continuing interest. This work has been supported in part by the National Aeronautics and Space Administration under contract NAS7-918 and grant NGR 05-002-160.
Acton, L. W., Catura, R. C., and Joki, E. G. 1975, Ap. J. (Letters), 195, L93. Armstrong, T. P., and Krimigis, S. M. 1971, J. Geophys. Res., 76, 4230.

Armstrong, T. P. Krimigis, S. M., Hovestadt, D., Klecker, B., and Gloeckler, G. 1976, Solar Phys., 49, 395.

Armstrong, T. P., Krimigis, S. M., Reames, D. V., and Fichtel, C. E. 1972, J. Geophys. Res., 77, 3607.

Bame, S. J., Asbridge, J. R., Feldman, W. C., Montgomery, M. D., and Kearney, P. D. 1975, Solar Phys., 43, 463.

Bertsch, D. L., Biswas, S., Fichtel, C. E., Pellerin, C. J., and Reames, D. V. 1973, Solar Phys., 31, 247.

Bertsch, D. L., Biswas, S., and Reames, D. V. 1974, Solar Phys., 39, 479.

Bertsch, D. L., Fichtel, C. E., and Reames, D. V. 1972, Ap. J., 171, 169.

Bertsch, D. L., and Reames, D. V. 1977, Solar Phys. 55 491.

Biswas, S., and Fichtel, C. E. 1965, Space Sci. Rev., 4, 709.

Boschler, P., and Geiss, J. 1976, Grenoble IAU Meeting, Report of Commission 12 (Solar Atmosphere).

Briggs, P. R., Armstrong, T. P., and Krimigis, S. M. 1979, Ap. J. (Letters), 228, L83.

Claas, W. J. 1951, Rech. Astr. Obs. Utrecht, 12, Part 1.

Coffey, H. E. (ed.) 1977-1978, Solar-Geophysical Data, Nos. 398-412 (Boulder: US Dept. of Commerce).

Cook, W. R. 1981, Ph.D. thesis, California Institute of Technology.

Cook, W. R., Stone, E. C., and Vogt, R. E. 1980, Ap. J. (Letters), 238, L97.

Cook, W. R., Stone, E. C., Vogt, R. E., Trainor, J. H., and Webber, W. R. 1979, Proc. 16th Internat. Cosmic Ray Conf. (Kyoto), 12, 265.

Crawford, H. J., Price, P. B., Cartwright, B. G., and Sullivan, J. D. 1975, Ap. J., 195, 213.

Dietrich, W. F., and Simpson, J. A. 1978, Ap. J. (Letters), 225, L41

Flower, D. R., and Nussbaumer, H. 1975, Astr. Ap., 39, 295.

Geiss, J. 1982, Space Sci. Rev., 33, 201.

Goldberg, L., Muller, E. A., and Aller, L. H. 1960, Ap. J. Suppl., 5, 1.

Heckman, H. H., Perkins, B. L., Simon, W. G., Smith, F. M., and Barkas, W. H. 1960, Phys. Rev., 117, 544.

Hovestadt. D. 1974, in Solar Wind Three, ed. C. T. Russell (Los Angeles: University of California), p. 2.

Hovestadt, D., Klecker, B., Vollmer, O., Gloeckler, G., and Fan, C. Y. 1975, Proc. 14th Internat. Cosmic Ray Conf. (Munich), 5, 1613.

Hurford, G. J., Mewaldt, R. A., Stone, E. C., and Vogt, R. E. 1975, Ap. J. (Letters), 201, L95.

Lambert, D. L. 1978, M.N.R.A.S., 182, 249.
Lambert, D. L., and Luck, R. E. 1978, M.N.R.A.S., 183, 79.

Mariska, J. T. $1980, A p . J ., 235,268$.

Mason, G. M., Hovestadt, D., and Gloeckler, G. 1979, Proc. 16th Internat. Cosmic Ray Conf. (Kyoto), 5, 110.

McGuire, R. E., von Rosenvinge, T. T., and McDonald, F. B. 1979, Proc. 16th Internat. Cosmic Ray Conf. (Kyoto), 5, 61.

Mewaldt, R. A. 1980, in Proc. Conf. Ancient Sun, ed. R. O. Pepin, J. A. Eddy and R. B. Merrill (New York: Pergamon Press), p. 81.

Meyer, J. P. 1979, in Les elements et leurs isotopes dans l'univers, 22nd Liège Internat. Astr. Symp. (University of Liège Press), p. 153.

. 1981a, Proc. 17th Internat. Cosmic Ray Conf. (Paris), 3, 145 1981b. Proc. 17th Internat. Cosmic Ray Conf. (Paris), 3, 149.

Meyer, J. P., and Reeves, H. 1977, Proc. 15th Internat. Cosmic Ray Conf. (Plovdiv), 2, 137.

Mogro-Campero, A., and Simpson, J. A. 1972, Ap. J. (Letters), 177, L37.

Nevatia, J., Durgaprasad, N., and Biswas, S. 1977, Proc. 15th Internat. Cosmic Ray Conf (Plovdiv), $5,48$.

O'Gallagher, J. J., Hovestadt, D., Klecker, B., Gloeckler, G., and Fan, C. Y. 1976, Ap. J. (Letters), 209, L97.

Parkinson, J. H. 1977, Astr. Ap., 57, 185.

Pellerin, C. J. 1975, Solar Phys., 41, 449.

Ross, J. E., and Aller, L. H. 1976, Science, 19, 1223

Russell, H. N. 1929, Ap. J., 70, 11.

Scholer, M., Hovestadt, D., Klecker, B., Gloeckler, G., and Fan, C. Y. 1978, J. Geophys. Res., 83, 3349.

Stone, E. C., Vogt, R. E. McDonald, F. B., Teegarden, B. J., Trainor, J. H., Jokipii, J. R., and Webber, W. R. 1977, Space Sci. Rev., 21, 355.

Teegarden, B. J., von Rosenvinge, T. T., and McDonald, F. B. 1973, Ap. J., 180,571 .

Van Allen, J. A., Venketarangan, P., and Venkatesan, D. 1974, J. Geophys. Res., 79, 1.

Veck, N. J., and Parkinson, J. H. 1981, M.N.R.A.S., 197, 41.

Webber, W. R. 1982, Ap. J., 255, 329.

Webber, W. R., Roelof, E. C., McDonald, F. B., Teegarden, B. J., and Trainor, J. 1975, Ap. J., 199, 482.

Wibberenz, G. 1979 Proc. 16th Internat Cosmic Ray Conf. (Kyoto), 14, 234.

Withbroe, G. L. 1975, Solar Phys., 45, 301.

Zwickl, R. D., Roelof, E. C., Gold, R. E., and Krimigis, S. M. 1978, Ap. J., 225, 281

W. R. Cook: 220-47 Downs Laboratory, California Institute of Technology, 1201 E. California, Pasadena, CA 91125

E. C. Stone: 103-33 E. Bridge Laboratory, California Institute of Technology, 1201 E. California, Pasadena, CA 91125

R. E. Vogt: Office of the Provost, 206 Parsons-Gates, California Institute of Technology, Pasadena, CA 91125 